\title{
Persiano, mago e guerriero. Note sulla caratterizzazione Di MANi E DEI MANICHEI NELLE FONTI GRECO-LATINE DEL IV SECOLO
}

\author{
Andrea Piras
}

Università di Bologna

\begin{abstract}
The perception of cultural otherness in Late Antiquity and within the Christian or pagan milieu shows a very interesting case of condemnation, about which these two sides of the Roman society were in agreement. The prophet Mani was in fact the target of a shared feeling of disapproval towards an alien faith strongly characterised by its "Persian" provenance. Greek and Latin authors, starting with the Neo-Platonic Alexander of Lycopolis, emphasised the links between Mani and the Persian Empire, where he lived, under the patronage of the king of kings Šābuhr I. The polemic against Mani and his dualistic doctrine, labelled as "heretical", was strengthened by means of added epithets such as "Persian", "Magician" and "Warlord", drawing upon a wellestablished repository of bias and stereotyped formulae belonging to the Roman and Hellenistic culture.
\end{abstract}

Key words: heretic, Persian, magician, warlord, Mithraism, histrionic behaviours, prejudices, stereotypes, Late Antique Roman mentalities.

La percezione della cultura classica ed ellenistica verso la potenza straniera dell'Iran, o della Persia - se si vuole scegliere la parte geografica eminente, sacrario dinastico di Achemenidi e Sassanidi -, ha elaborato linguaggi e codici identificativi di ampio spettro semantico, per designare un secolare vicino invadente, anche se con una cultura e in specie con una ricchezza tali da suscitare avidità mista a invidia ma anche rispetto e ammirazione. La storia culturale e ideologica di questi "sguardi verso oriente", da parte di un osservatore giustamente timoroso di tale potenza imperiale, attiva e minacciosa, è quella registrata in una estesa documentazione greca e latina che ci ha restituito molti dati; anche se, inevitabilmente, nello specchio deformante del soggetto autoptico, a sua volta non neutrale verso l'informazione acquisita che lo portava a "inventare il barbaro", 1 e segnatamente a "inventare la Persia", per definire sia l'Altro sia la propria difesa auto-identitaria. Purtroppo non abbiamo, da parte iranica, una analoga messe di

\footnotetext{
${ }^{1}$ Mi riferisco qui al capitolo "Inventing Persia" del libro di Hall 1989, 56-100.
} 
dati o di curiosità verso un mondo occidentale limitrofo o intersecante a vario titolo (scambi, commerci, soldati mercenari): e questo a dispetto della attitudine assimilatoria dei Persiani verso altre culture e società, come ci ricorda Erodoto (I, 135) in un celebre passo. Questo dipende dal fatto che la investigazione storica necessita di altri presupposti che la annalistica orientale, sottoposta al vaglio del Gran Re, non esaudiva, ${ }^{2}$ non prevedendo individualità autoriali come quelle del mondo ellenico.

I colleghi intervenuti in questi lavori congressuali hanno già anticipato argomenti che preparano le mie osservazioni, a seguito dell'esame di autori come Strabone, Plutarco, Tacito e Ammiano; oppure inoltrandosi nelle terrae incognitae delle steppe scitiche, lambite dalla grecità periferica; o ancora, in quell'ambito bizzarro e controverso del mithraismo, culto di un mondo romano, ostile sì alla Persia ma che paradossalmente, nella gerarchia iniziatica dei suoi misteri poteva assumere il grado mistico di "Persiano". Il mio ambito verterà su un proseguimento di questo "sguardo verso oriente" in una diversa situazione culturale e religiosa: quella di una cristianità del IV secolo, gradualmente trionfante, e dei suoi nemici confessionali, gli eretici, gnostici o manichei che fossero. Contro questi ultimi, i manichei, veniva indirizzato, in un caso specifico, un linguaggio carico di una secolare tradizione di sospetto e di pregiudizi verso una origine straniera (persiana) nefasta e minacciosa verso un ordine istituzionale che richiedeva meccanismi protettivi, anche nella sfera pubblicistica, verbale e oratoria della parola e della confutazione retorica. Gli scenari pubblici della disputa, ${ }^{3}$ per il consenso nella dottrina filosofica o religiosa, sono quelli che meglio illustrano le competizioni dell'eloquenza per isolare e sconfiggere l'avversario, con lo stigma della riprovazione e della condanna, o con la denuncia della inconsistenza razionale e argomentativa della sua dottrina.

Il caso che prenderò in esame riguarda una categoria socio-religiosa, quella dei manichei, che venne sanzionata non tanto per la generica attribuzione della etichetta ereticale, quanto, in sovrappiù, per essere stati i destinatari di una duplice ostilità, prima del mondo romano-pagano e poi di quello romano-cristiano. La distinzione non è di poco conto e ci permette di cogliere un supplemento e un'enfasi di biasimo e di condanna che ha veicolato contro questa religione sia la aggressività imperiale tetrarchica sia quella ecclesiastica; e infine quella degli imperatori cristiani, a partire dalle vicende che aprirono al cristianesimo il favore di Roma, dopo Costantino e ancor più dopo Teodosio. In questa dirompente miscela di stereotipi, di mentalità e di codici, sia politici che confessionali, noteremo che l'efficacia della condanna poteva fondarsi su una consuetudine plurisecolare di discorsi contro i "barbari" persiani che si ripresentavano ora, in modo ancora più insidioso, nelle ingannevoli vesti della eresia.

Gli studi sul manicheismo e sul più ampio insieme dello gnosticismo, a cui appartiene, vantano una consolidata dottrina di edizioni di testi ${ }^{4}$ e di studi, nelle disparate lingue in cui tale religione si espresse, in conformità alla sua vocazione missionaria

${ }^{2}$ Cf. Klíma 1968, 219-220, per tali considerazioni sull'aspetto soggettivo e autoriale della storiografia greca, contro la più anonima annalistica persiana.

${ }^{3}$ Sulle strategie retoriche della costruzione di un linguaggio anti-ereticale in età tardo antica cf. Cameron 2013, 7-12.

${ }^{4}$ Rimando alla traduzione italiana di testi manichei curata da Gnoli, con l'assistenza di Piras 2003; 2006; 2008 . 
e multilingue (greco, copto, latino, iranico [medio-persiano, partico, sogdiano, battriano], turco, tochario, cinese). A tali fonti primarie si aggiunga una ricca letteratura polemistica ed eresiologica di fonti secondarie (arabe, siriache, greche, latine). In tale contesto, ci baseremo unicamente sulle fonti di confutazione greche ${ }^{5}$ e latine, per cogliere quella continuità di stereotipi che la cultura romana elaborò, traendoli dal più antico patrimonio classico della grecità e della ostilità contro la Persia, ereditata da Roma ${ }^{6}$ attraverso la mediazione di Alessandro, exemplum di imitazione che trasferiva su Roma tutto un lascito antico di valori, linguaggi e scelte comportamentali.

A partire dalle testimonianze letterarie del III secolo si registrano delle notizie che configurano una percezione variabile della dottrina manichea: da una parte, vi è il primo documento antimanicheo, la lettera pastorale del vescovo Theonas di Alessandria ( \pm 280$)$ - ovvero il Papiro Rylands $469^{7}$ - che ammoniva i fedeli contro il culto dei manichei e la loro avversione per il matrimonio; e dall'altra parte, vi è l'opera di un altro egiziano, Alessandro di Licopoli $( \pm 290)$, filosofo neoplatonico che ospitò nella sua scuola diversi manichei e che ci ha lasciato una valutazione più serena, benchè critica in vari punti, ma senza i rigori confessionali dei cristiani, e in più, preziosa per un sommario della dottrina manichea da lui fornito. ${ }^{8}$ La documentazione del IV secolo fu invece non solo più ingente ma più caratterizzata in senso confessionale, a partire da Eusebio di Cesarea (301-302) nella sua Storia Ecclesiastica (VII, 31), a Cirillo di Gerusalemme (348) nella sua quarta Catechesi battesimale, a Serapione di Thmuis (morto nel 358), ad Epifanio (376) e al suo Panarion (eresia 66), fino all'opera di Agostino che dal 388/389 (data del De moribus Manichaeorum) agli inizi del V secolo scrive le sue opere antimanichee. A ciò si aggiunga la legislazione imperiale di Valentiniano I (372) e di Teodosio I (380) che fissano i capisaldi giuridici di un attento controllo socio-politico e religioso (interdizioni, confische, pena di morte) che si inasprirà poi con le legislazioni giustinianee del VI secolo. ${ }^{9}$

All'interno di questa vivace produzione letteraria, apologetica e militante, l'opera ${ }^{10}$ degli Acta Archelai attribuiti ad Egemonio, più o meno composta verso il 340 (la versione greca; quella latina, nel 365), presenta - oltre ai dati storici e geografici - un ritratto della vicenda esistenziale di Mani un po' romanzata ma di estremo interesse; a cui si aggiunga un affidabile resoconto dottrinale del sistema di Mani che servì da modello per la posteriore letteratura eresiologica e polemistica. ${ }^{11}$ I passi in cui si descrive l'arrivo di Mani nell'ambiente cittadino, attorniato da una schiera di devoti, ci attestano un riuscito mélange di realtà e di finzione, nella caratterizzazione negativa del personaggio, in cui

${ }^{5}$ Una raccolta di testi prodotti nell'impero romano è quella di Gardner - Lieu 2004. Per gli originali greci e latini cf. Adam 1969 e il più recente Fox - Sheldon - Lieu 2010 (testi originali, traduzioni e commenti). Sul manicheismo nell'impero romano cf. Lieu 1999, 22-131.

${ }^{6}$ Per le ripercussioni delle guerre greco-persiane nella cultura romana cf. Spawforth 1994 e Vannicelli 2007, 592-597. Sull'elemento persiano nella letteratura latina, da Plauto a Ammiano, si rimanda al sempre attuale saggio di Paratore 1966.

7 Testo in Adam 1969, 51-54; traduzione in Gardner - Lieu 2004, 114-115.

${ }^{8}$ Per uno studio di quest'opera di Alessandro di Licopoli (il Contra manichaei opiniones disputatio), cf. Villey 1985.

${ }_{9}$ Per una cronografia di autori e opere cf. Tardieu 1981, 112-115.

${ }^{10}$ Per l'edizione, cf. Beeson 1906. Traduzione e commento in Vermes - Lieu - Katz 2001.

${ }^{11}$ Come nel Panarion (25-31) di Epifanio, che ne riporta l'estratto dottrinale: cf. Riggi 1967, 110-152. 
si sommano, efficacemente, alcuni stereotipi della cultura ellenistica contro 1'Altro (il nemico, prima politico e poi religioso), originario dalla Persia.

Prima di esaminare l'opera consideriamo alcuni retroscena. Il tempo che intercorre, retroattivamente, fra la datazione di queste due versioni degli Acta Archelai (340 e 365) e la morte di Mani (276) si estende in una lunga gestazione di quasi un secolo (considerando la redazione latina), durante la quale la fama (e l'infamia) di Mani si propagò nel Mediterraneo romano, ingenerando dinamiche di sospetto, diffidenza e condanna: specialmente per delle connessioni tra Mani e la Persia che dovettero insospettire le autorità imperiali romane, da lungo tempo occupate a fronteggiare l'Iran partico e poi sassanide lungo il confine del limes mesopotamico.

Dobbiamo ad Alessandro di Licopoli, ${ }^{12}$ verso la fine del III secolo (290: la morte di Mani è databile al 276/277), il primo riferimento che connette Mani alla Persia e al re

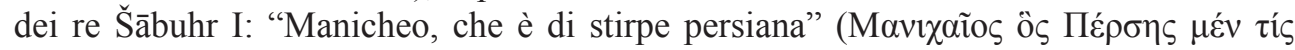

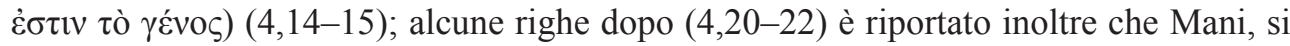

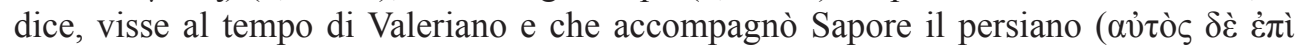

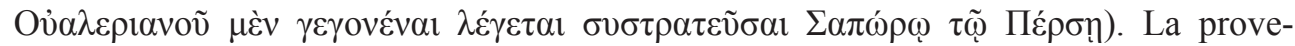
nienza egiziana (Licopoli, nell'Alto Egitto) di questa importante notizia della fine del III secolo ci è utile per comprendere la reazione ostile dei Tetrarchi, a partire da Diocleziano e dal suo rescritto contro i manichei (31 marzo 302) inviato da Alessandria al proconsole d'Africa, che inaugurò un clima di aspra condanna e repressione, i cui effetti si propagarono nella legislazione delle epoche successive, al punto da essere norma giuridica che veniva ricordata nell'impero cristiano per legittimare l'autorità dei sovrani. Una recente opera di Umberto Roberto ${ }^{13}$ dedicata a Diocleziano riassume il clima e le avvisaglie che alla fine del III secolo e delle lotte tra i Soldatenkaiser portarono Diocleziano e le istituzioni tetrarchiche a codificare una linea di repressione contro quella Persica adversaria nobis gens che per molti decenni del III secolo aveva messo a dura prova gli eserciti romani, ${ }^{14}$ a partire dall'esordio della dinastia sassanide, con Ardašīr, e con le vittorie di Šābuhr I contro Valeriano, fino a quella situazione di ribaltamento, sotto Narseh e per merito degli sforzi di Galerio, culminata nella tregua del 298 favorevole ai romani. ${ }^{15}$

Nonostante questo esito positivo e comunque momentaneo, date le oscillazioni tra guerra e tregua che caratterizzavano il rapporto tra le due superpotenze, Diocleziano vigilava con accorta supervisione su ogni possibile scintilla di rivolta e disordine: come infatti percepì nei rapporti su questa infame secta e sui suoi legami con il mondo persiano, almeno nei termini di provenienza (il che non comportava affatto, anzi al contrario, che i manichei fossero gli emissari della Persia). In ogni caso, se la notizia di Alessandro poteva essersi diffusa, la coincidenza con Valeriano più l'abbinamento con la Persia e con il re dei re Šābuhr I sarebbero bastate a provocare attenzioni guardinghe della autorità: quindi a promuovere misure di deterrenza e di repressione contro le scaevas leges Persarum e la doctrinam Persarum, abbinata a pratiche oscure e stregonesche, come si

\footnotetext{
12 Testo greco in Adam 1969, 54.

${ }_{13}$ Roberto 2014a, 189-194. Vedi anche Roberto 2014b. Per il testo dell'editto di Diocleziano, cf. Adam 1969, 82-83.

${ }^{14}$ Sulle guerre tra Roma e Persia nel III secolo, cf. Gnoli 1996; Dignas - Winter 2007, 18-32. Cf. ora sulla disfatta di Valeriano, Coloru 2017.

${ }^{15}$ Su questa tregua fra Narseh e Galerio, e sul rifiuto della isotimia verso la Persia, cf. Roberto 2014b, 186-195.
} 
evince dalla titolazione dell'editto (De mathematicis, maleficis et Manichaeis), a riprova di una connessione con i maleficia sempre efficace per fomentare il biasimo e la persecuzione nel mondo romano. ${ }^{16}$

Le severe prescrizioni tetrarchiche saranno quindi normative per i tempi futuri; e la pubblicistica, a partire da Alessandro di Licopoli, a Eusebio, a Teodoreto, Agostino (fino al lessico $S u d a$ ), contribuirà a drammatizzare i toni del confronto, saldando inestricabilmente Mani alla venefica terra di Persia. ${ }^{17}$ Una prudente e circospetta attitudine di strategia e di informazione si registra non solo in epoca tetrarchica ma anche in quella costantiniana, sia per mantenere la sicurezza dei confini sia per oltrepassarlo, in eventuali scenari di guerra, come avvenne con la progettata campagna persiana di Costantino, che però non ebbe luogo a causa della sua morte (337). Vero è che a parte questa spedizione inadempiuta, il pericolo persiano era diffuso nell'epoca di Costantino, anche se stemperato dalle precedenti vittorie tetrarchiche, e sebbene convivente con tendenze più innocue di moda e di importazione di oggetti orientali di lusso (vesti) o di decoro regale (elmo diademato). ${ }^{18}$ In un caso specifico, un clima di insinuazioni e di accuse, rinfocolato dal sospetto che le usanze persiane (come quelle dei matrimoni consanguinei ${ }^{19}$ ) potessero infettare gli austeri costumi romani, fu all'origine della triste vicenda del figlio di Costantino, Crispo (nato dalla concubina Minervina), da lui fatto uccidere nel 326 per fugare i sospetti di una sua presunta relazione con la moglie Fausta; sospetti che in modo del tutto capzioso e dissimulatorio Giuliano dovette, parimenti, suggerire qualche tempo dopo, nella sua orazione per Costanzo (I, 9), e nel parallelo contrastivo tra la virtù di Fausta e l'immoralità incestuosa di Parisatide. ${ }^{20}$ In tale caso, il sospetto di immoralità nei costumi sessuali e matrimoniali - un topos di alterità sociale che insieme alle deprecate usanze funerarie si ritroverà nella pubblicistica tardo-antica, fino ad Agazia ${ }^{21}$ - costituì per Costantino una insidia non certo manichea, ma anzi zoroastriana, data la continenza e l'avversità dei manichei per il matrimonio: a differenza degli zoroastriani, per i quali il matrimonio, e in specie quello consanguineo, era espressione della bontà del dio creatore Ohrmazd in un mondo dove le energie procreative (prole, agricoltura, allevamento) affermavano la vita contro la negatività della morte, della sterilità e della carestia. È vero, al contrario, che Costantino manifestò un certo interesse strategico per i manichei, forse anche per esigenze di controllo in quei territori limitrofi (Palmira, regno arabo di al-Ḥira), di interfaccia con l'impero sassanide, in cui la loro espansione missionaria li conduceva, in peregrinazioni religiose che avrebbero potuto comportare attività informative (spionistiche) di una qualche utilità. ${ }^{22}$ Ma di questa propensione cautelativa di Costantino

16 Cf. Barb 1975, 127.

${ }^{17}$ Cf. i testi e le traduzioni commentate in Fox - Sheldon - Lieu 2010, 43 (Eusebio), 85 (Agostino), 93 (Teodoreto), 129 (Suda).

18 Si vedano le considerazioni sull'ambiente e la società di Costantino in Piras 2013, 416-420.

19 Per una disamina sugli autori classici che menzionano questa forma di matrimonio, cf. Bucci 1978.

${ }^{20}$ Cf. le giuste osservazioni di Marasco 1993, 315-316. E vedi Tantillo 1997, 65, 194-196, per l'orazione a Costanzo.

21 Si rimanda a Questa 1989, per una trattazione della ideologia funeraria e matrimoniale (incesto) dei Persiani, e per la loro ricezione (e ricusazione, per supposta inciviltà barbarica) nell'opera di Agazia.

22 L'incarico conferito da Costantino al funzionario Strategio Musonio, di investigare sui manichei, è un aspetto che chiarisce questa attenzione imperiale: cf. Minale 2013 e 2014, anche per la bibliografia e il relativo dibattito. 
non ne è decifrabile la traccia negli Acta Archelai, dove senza distinzioni si affastellano clichés, pregiudizi e sommarie caratterizzazioni delle usanze "barbariche", in un'epoca di transito fra la cultura pagana e quella cristiana che la nostra opera testimonia e che le premesse fin qui esposte possono aiutarci a comprendere.

In quanto opera composita, di informazioni e di finzioni, il testo degli Acta Archelai ha costituito materia di analisi in importanti contributi e convegni. ${ }^{23}$ Nella versione latina di Acta Archelai XIV, dopo aver menzionato Mani che portava con sé fino a ventidue Eletti, giovani e vergini (Manes adducens secum iuvenes et virgines electos ad viginti $d u o)$, il protagonista del racconto, Marcello, descrive il suo abbigliamento con un certo stupore (admiratus est primo habitus indumenta). L'impressione del suo aspetto e dei suoi vestiti è tale infatti da sintetizzare alcuni tratti emblematici di una figura di alienità e di estraneità culturale:

aveva un tipo di calzatura che la gente era solita chiamare trisolium; e un mantello variopinto, quasi fosse di una parvenza eterea; in mano teneva invero un bastone molto resistente, di legno d'ebano; portava sotto il braccio sinistro un libro babilonese e aveva le gambe coperte da pantaloni di diversi colori, una di colore rosso e l'altra come di colore verde. Il suo aspetto sembrava davvero quello di un vecchio mago persiano o di un comandante

habebat enim calciamenti genus quod trisolium vulgo appellari solet; pallium autem varium, tamquam aërina specie; in manu vero validissimum baculum tenebat ex ligno ebelino; Babylonium vero librum portabat sub sinistra ala; crura etiam bracis obtexerat colore diverso, quarum una rufa, alia velut prasini coloris erat. Vultus vero ut senis Persae artificis et bellorum ducis videbatur. (Acta Archelai XIV, 2; ed. Beeson 1906, 22)

Questa raffigurazione, per i vari clichés che disegnano un'effigie satirica, è stata più volte commentata. Si è anche proposto di valutarla come plausibile ritratto standard di Mani che poteva riflettersi anche in testimonianze figurative, come un mosaico del IV secolo, di provenienza siriaca, dove un personaggio con berretto floscio porta un libro e un bastone. ${ }^{24} \mathrm{E}$ questo spiegherebbe ulteriormente l'efficacia comunicativa di tale descrizione topica, recepita dagli stessi Acta Archelai, e che rispondeva a un codice iconicotestuale diffuso nel Levante e nel Medio Oriente. Tra le varie interpretazioni, l'esegesi più acuta e circostanziata di questo passo è quella di un recente contributo di Scopello ${ }^{25}$ che ha ricostruito lo sfondo storico-culturale e letterario degli Acta Archelai proprio in relazione a questa descrizione di Mani, in cui si saldano diverse suggestioni e pregiudizi relativi al nemico, non solo etnico-politico e di alienità linguistica ("caldeo") ma anche religioso. In tale modo, l'accusa di eresia (devianza confessionale eterodossa) rispetto a statuti emergenti di ortodossia e intransigenza, si intreccia - come vedremo più sotto - con quella di paganesimo mithraico di culti solari (e romano-imperiali) officiati con risibili e deprecabili comportamenti dell'ambiente teatrale. L'acuta disamina di Scopello coglie degli importanti nessi tematici di propaganda e di sconfessione dell'avversario dottrinale, insistendo sull'abbigliamento scenico, come rivelato dal trisolium, scarpa con rialzo usata dai teatranti - ma anche mezzo ortopedico adatto per la zoppaggine, una invalidità forse

${ }^{23}$ Rimando agli atti congressuali del volume curato da BeDuhn - Mirecki 2007, anche per ogni trattazione pregressa.

${ }^{24}$ Il mosaico (presente nel Bible Lands Museum di Gerusalemme) è stato studiato da Lieu 2000, 383-385.

${ }^{25}$ Scopello 2008, 161-164. 
congenita a Mani, su cui ci si è spesso soffermati, anche per cogliere dei possibili risvolti psicologici della sua indole (introspezione, fuga dal mondo, misticismo). ${ }^{26} \mathrm{~A}$ completamento della ipotesi di Scopello, ho proposto che il trisolium rinviasse alla infermità, da intendersi anche come contrassegno di una alterità e marginalità che poneva Mani nel rango di quei personaggi mitici, eroici (Edipo, Melampo, Giasone, Perseo) o divini (Hermes "monocrepide"), dotati di carismi "liminali" per transitare (camminare) tra questo mondo e l'Aldilà, mediante deambulazioni asimmetriche e oblique, come appunto suggerito da una condizione claudicante o di monosandalismo.

Rispetto a Scopello, questa mia interpretazione vuole essere corroborante e non certo antitetica. Entrambe colgono la "marginalità" del soggetto, il cui retroterra mitico (anche se di un immaginario semitico-orientale e non certo greco-ellenistico) viene obliterato dai significanti teatrali che Scopello ha ben messo in risalto e che denotano una caricatura, nel segno dell'irrisione e del biasimo, confacente a quell'odium theologicum che uno studio di Klíma ${ }^{27}$ riconduceva ad analoghi intenti di scherno e diffamazione: tramite una narrazione intrisa di elementi classici ma ribaltati, negati nelle loro valenze originarie e anzi ridicolizzati secondo i nuovi codici del linguaggio cristiano. In tale modo, Mani l'artifex nel senso di "artista" (Künstler) che calza il trisolium, munito di bastone e accompagnato da un suo seguito, poteva ricordare l'Efesto omerico (Iliade

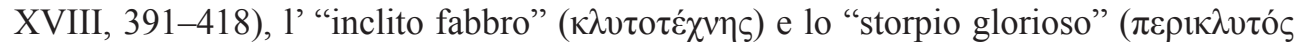

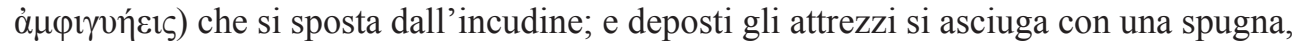
veste la tunica e preso un grosso bastone esce dal suo antro zoppicando, aiutato da due ancelle che lo sostengono. Il patrimonio culturale e mitologico della cultura ellenistica poteva trovare inediti sbocchi di invenzione narrativa, per connotare negativamente avversari quali Mani (persiano, eretico, mago), creatore di nuove fabulae mitologiche, residui pagani trattati con sarcasmo e disprezzo dagli intellettuali tardo antichi. Questo dispositivo letterario di biasimo e di manipolazione retorica del linguaggio poteva esprimere nella parodia e nei virtuosismi paraetimologici l'odium theologicum, in giochi linguistici tra significanti e significati: come quelli tra Paraclito ( $\pi \alpha \rho \alpha ́ \kappa \lambda \eta \tau o \varsigma)$, epiteto che Mani si attribuiva, e $\pi \alpha \rho \alpha ́ \pi \lambda \eta \kappa \tau o \varsigma$ ("pazzo, demente"): procedimento uguale a quello applicato al nome proprio semitico (aramaico) di Mani, distorto in varie forme che rinviavano per assonanza alla "follia" (Mani < maneis) e quindi all'insania di una dottrina perversa, frutto di dissennatezza e di devianza intellettuale. ${ }^{28}$

Il bastone non viene fatto oggetto di manipolazioni a scopo satirico: poteva indicare un tipo di insegna del pellegrino e della guida di un "gregge" di fedeli, simile quindi a un pastorale o a un'analoga insegna di autorità sapienziale, cui non doveva essere estraneo anche un uso apotropaico ed esorcistico. ${ }^{29} \mathrm{E}$ in quanto emblema di filosofi e maestri itineranti, il bastone poteva essere spesso menzionato in vari contesti interconfessionali della spiritualità tardo antica. ${ }^{30} \mathrm{Al}$ contrario, i vestiti sono invece più caratterizzati in

${ }^{26}$ Per quanto segue, cf. Piras 2005. Il tema mitico della zoppaggine e del monosandalismo è stato ampiamente trattato da Ginzburg 1995, 207-237.

27 Klíma 1957, 385.

${ }_{28}$ Cf. le analisi di Coyle 2004, 221-223 sulla mania applicata al nome di Mani.

${ }^{29}$ Le osservazioni di Gignoux $(1993,65-67)$ riconducono questa insegna a un più esteso ambiente culturale, sia manicheo, che cristiano o zoroastriano.

30 Cf. Brown 1999, 661-663, 673. 
modo ideologico e critico. Sia il mantello (pallium) che i calzoni (bracae) sono indicatori di una alterità di costumi (bracatus era il forestiero e quindi il barbaro), resa ancora più intensa dall'uso dei colori, sia per il mantello che per i calzoni, con un'enfasi che denuncia nel variopinto (varium) e nella duplice colorazione delle bracae un sovrappiù di ripulsa verso una poikilia che i codici estetici dell'abbigliamento cristiano, nelle prescrizioni dei Padri della Chiesa, denunceranno come marchio di estraneità alle convenzioni sociali e al preferibile colore uniforme dell'abbigliamento sobrio e grezzo, di cotone o di lino. ${ }^{31}$ Di nuovo, la variopinta ricchezza dell'abbigliamento orientale rinviava a una lunga trafila di autori ${ }^{32}$ che nelle loro descrizioni dell'abito straniero potevano evidenziare il rischio di incaute assunzioni di vesti (e quindi di habitus comportamentale) e di tratti "medizzanti", ${ }^{33}$ forieri di cambiamenti di attitudini considerate riprovevoli. L'esempio più antico di Pausania (Tucidide I, 129-130), che dopo aver letto la missiva di Serse assume il manto persiano e si circonda di una guardia del corpo persiana, rendendosi inaccessibile, è un precedente che poteva riverberarsi nella storia delle convenzioni sociali, estetiche e culturali dall'antichità classica a quella tardo-antica. L'impiego di questi tratti identificativi, stratificati in una lunga memoria di tradizioni documentarie, sortisce il suo effetto sul vescovo Archelao: che a causa dell'abito e dell'aspetto di Mani (ex ipso habitu ac specie eius) decide di intraprendere la disputa contro di lui (XIV, 4).

Oltre all'abbigliamento, va considerato il discredito che si estende alla parlata barbara del persiano (Acta Archelai XL) che non si è istruito in alcuna altra lingua dell'ecumene mediterranea greca, romana (e addirittura egiziana) ma solo in quella dei Caldei (Persa barbare! Non Graecorum linguae, non Aegyptiorum, non Romanorum, non ullius alterius linguae scientiam habere potuisti, sed Chaldeorum solam). La etichetta di "caldeo" può denotare varie intenzioni, sia per la nota tradizione sapienziale mesopotamica (cui allude il Babylonium librum), sia per i luoghi dove sono ambientati i contesti degli Acta Archelai e della citta della "Caldea", Charcar, in cui si svolge la disputa con Mani e dove si situa la sua apparizione variopinta e il suo corteo di fedeli. Ciò corrisponde, del resto, a una effettiva identità culturale di Mani stesso che in diversi scritti rivendicò sempre la sua provenienza dalla terra di Babilonia. Ma per un pubblico romano, il termine "caldeo", se avvicinato all'espressione artifex sopra incontrata, caratterizzava geograficamente quei maghi la cui sapienza mesopotamica ("caldea") di Magusei o di "Magi ellenizzati", a partire dalla conquista di Alessandro (ma iniziata ben prima, nell'impero achemenide, quando i magi si diffusero in Mesopotamia), denotava quelle competenze magiche e divinatorie verso cui la cultura classico-ellenistica ebbe sempre una propensione duplice: in parte estimativa e in parte repulsiva, per quell'alienità di una "sapienza straniera" che univa la rispettabile "terapia delle

31 Cf. Neri 2004, 226.

32 Sull'abbigliamento persiano, cf. Senofonte, Anabasi I, 5, 8: soprabiti di porpora, tuniche, calzoni vari-

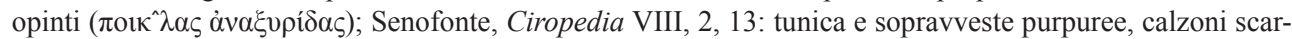
latti. Arriano, Anabasi, VI, 29, 4-8: vesti tinte di viola e di porpora (tomba di Ciro ). Curzio Rufo III, 3, 13: vesti trapunte d'oro, tuniche con maniche lunghe, adorne di gemme (abito degli "Immortali"); III, 3, 17-18: tunica purpurea e mantello dorato (Dario III).

33 Cf. Graf 1984, 15 su Pausania. Sulle ambivalenze del termine "medismo" (scelta politica o predilezione estetica per la moda orientale), cf. le giuste osservazioni di Tuplin 1997, 180-185. 
cose divine", della magéia, a quelle pratiche screditate della goetéia e delle sue imposture..$^{34}$ Un "persiano" artifex, per giunta "caldeo" ed esprimentesi in "caldeo" (cioè in aramaico: in effetti, la lingua di Mani), poteva raffigurare un emblema suggestivo dalle molteplici significazioni, evocative di antichi e contemporanei pericoli. A ciò si aggiunga che il tempo della redazione greca (340) e latina dell'opera (365) si situa in quel periodo che vide sorgere la persecuzione dei cristiani nell'impero sassanide, promossa dal re dei re Šābuhr II fra il 339 e il 379, non solo dentro le zone geografiche dell'Iran ma in quelle terre della Babilonia e della Mesopotamia in cui si situa la nostra opera. Non è quindi inverosimile che i critici eventi dell'epoca, di natura sia militare (mancata conquista di Nisibi, sconfitta dei persiani a Singara) che politica ed economica (massiccia esazione di imposte e doppio tributo imposto ai cristiani ma rifiutato dal Katholikos), insieme a quelle situazioni di deportazioni e riscatto di prigionieri, ${ }^{35}$ da ambo le parti del limes e che sono menzionate negli Acta, possano aver influito sul clima degli ambienti e della mentalità del tempo, inclusa quella dell'autore di un'opera i cui luoghi mesopotamici erano pervasi da conflitti e tensioni. Anche se la evocazione letteraria del persiano Mani artifex che parla la Chaldeorum lingua non corrisponde totalmente al sacerdote della casta dei magi zoroastriani - che in effetti lo perseguitarono: ma ciò non poteva essere noto a Egemonio e ad altri - tuttavia le sue caratteristiche esteriori, in questo mélange guerriero e stregonesco, lo ricordano e ne suggeriscono le funeste implicazioni, nocive alla cultura e alla società romana, prima pagana e poi cristiana. Sicuramente, i cristiani dovevano avere una qualche idea dello zelo persecutorio dello zoroastrismo sassanide, come ci testimoniano le iscrizioni del gran sacerdote Kirdīr (III secolo), dove in un noto passo si compiace di aver colpito le minoranze religiose dell'impero, fra le quali i cristiani, nella loro duplice qualifica di cristiani dentro e fuori l'ecumene sassanide. A ciò si aggiunga che una rappresentanza di magi zoroastriani non doveva certo mancare nei quadri dirigenti degli eserciti persiani. L'istrionico maguseo, dietro il cui sembiante si tratteggia Mani il persiano, viene forse qui evocato in rappresentanza di una categoria sacerdotale che nella storia dell'impero sassanide avrebbe promosso e incentivato una azione repressiva di lunga durata. ${ }^{36}$ Magi zoroastriani in veste di magusei e subdoli maghi artifices si sovrappongono in una descrizione del nemico atavico e della sua perniciosa "Alien wisdom".

La tipologia dei magusei non è comunque l'unica connotazione sacerdotale negativa che viene suggerita negli Acta Archelai. Al culmine di tutti questi epiteti troviamo infatti la qualifica di sacerdote di Mithra (O barbare sacerdos Mithrae) e del suo culto misterico solare (solem tantum coles Mithram locorum mysteriorum inluminatorem) che a detta di Egemonio Mani amministra, di nuovo, con quel fare istrionico (tanquam elegantem mimum perages mysteria) che lo ridicolizza ulteriormente agli occhi di un pubblico austero e cristiano, per la deplorazione verso la teatralità pagana e le sue maschere che assommano futilità e infingimenti, disdicevoli per la morale della nuova civitas. L'opera coglie, nella sua derisione caricaturale, alcuni aspetti della religione di Mani che in virtù della sua mistica della luminosità è stata, in effetti, avvicinata a tratti

${ }^{34}$ Sui rapporti tra magi zoroastriani, "magia", "Caldei” e mondo greco, cf. Panaino 2010, 51-62; 2011, $361-364$

${ }^{35}$ Scopello 2008, 153-159.

${ }^{36}$ Su tutto ciò cf. Panaino 2004, 799-807, e più in generale tutto il contributo. 
mithriaci ${ }^{37}$ presenti nel sistema manicheo e nella sua intricata mitologia, che presenta una entità demiurgica, il Terzo Inviato, con alcune tipologie mithriache anche se non nel senso misterico de termine (quanto piuttosto, zoroastriano). Ma tutto ciò doveva essere sconosciuto all'estensore dell'opera, Egemonio, che purtuttavia ha mescolato con verisimile efficacia diverse componenti della eterogenea cultura mesopotamica del IV secolo. Non è escluso che il ricorso al "barbaro sacerdote di Mithra" abbia voluto esprimere, da parte cristiana, un accenno polemico alla divinità cara a Giuliano (morto nel 363), cultore dell'ancestrale paganesimo e promotore di una sua risorgenza, tra i cui dèi solari figurava anche quel Mithra che Giuliano ricorda nella sua Orazione a Elios re 41 (155b). La sfortunata campagna di Giuliano contro le armate persiane di Šābuhr II, e la ignominiosa pace del trattato che ne seguì nello stesso anno, fu certo una sconfitta romana ma anche un monito per quel residuo paganesimo (solare e mithriaco) che ancora non accettava pienamente la vittoria di un cristianesimo a cui gli Acta Archelai danno voce e testo, addensando in un grumo di immagini efficaci tutte le possibili etichette di antagonismo culturale e di reazione a una alterità storico-geografica e religiosa. Il bersaglio degli Acta, Mani l'eresiarca e sacerdote di Mithra, racchiude in sé i due estremi di una polemica cristiana, contro la devianza eterodossa e contro il paganesimo dei culti solari e mithriaci.

L'ultimo appellativo di $d u x$ bellorum è sicuramente il più improprio, se si considera l'indole ascetica di Mani. Ma l'efficacia delle stereotipo, come è noto, non richiede mai la attinenza storica, e anzi funziona indipendentemente da essa, nel meccanismo dell'allusione e della verosimiglianza. In parte spiegabile con le origini di Mani, effettivamente nato nell'impero arsacide (da nobile lignaggio materno) e cresciuto in quello sassanide, tra gli iniziali favori del re dei re Šābuhr I e di suo fratello Ohrmizd, sino alla sua disgrazia sotto Wahrām I, complice la ostilità del gran sacerdote Kirdīr, promotore di un consolidamento del mazdeismo sassanide in chiesa di stato. Nonostante la sua sventura di condanna, incarcerazione e morte, e di estraneità all'establishment sassanide in quanto tale, la sua partecipazione al comitatus di Šābuhr I, ${ }^{38}$ durante la spedizione del re dei re contro Gordiano III, deve forse aver incrementato la sua fama di "Persiano", nei ranghi dei saggi e dei consiglieri di corte: ma certo non dell'esercito, data la natura pacifica e non-violenta della sua dottrina, similmente a quanto avvenne per il filosofo Plotino, un intellettuale alla ricerca della sapienza orientale che accompagnava Gordiano III, nei quadri dell'amministrazione e non certo in assetto di truce legionario. ${ }^{39}$ L'epiteto di dux bellorum fu di certo veicolato dall'abbigliamento sopra descritto (mantello, calzoni, berretto), uno stereotipo di vestiario "barbarico" comune nell'immaginario dell'orientalismo romano-ellenistico, da Augusto a Giustiniano, ${ }^{40} \mathrm{che}$ evocava la temibile potenza iranica, funestamente nota a Roma, a partire dalla sconfitta di Carre ${ }^{41}$ fino a una lunga sequenza di altri eventi bellici (sconfitta di Valeriano) o di negoziazioni umilianti: come la "ignominiosa pace" tra Gioviano e Šābuhr II (363) che

37 Ries 1979.

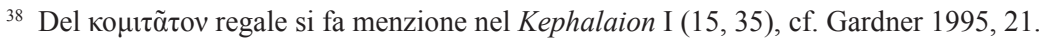

39 Sulla presenza di Plotino nelle armate di Gordiano III, cf. di recente Magris 2016, 30.

40 Sui codici di abbigliamento, riconoscibili da un vasto pubblico, e sul tema propagandistico dell'omaggio dei barbari vinti, nell'immaginario artistico dell'orientalismo romano, cf. Schneider 2006.

${ }^{41}$ Per Carre e le sue ripercussioni, cf. Traina 2010, 96-104 e 116-134; cf. anche Muccioli 2012. 
sottraeva a Roma le regioni transtigritane, e ridefiniva le aree di appartenenza iranica e di non ingerenza romana sull'Armenia. ${ }^{42}$ La versione latina degli Acta Archelai (riconducibile al 365) riflette, così pare, gli echi di una situazione critica e di una diffusa emozione collettiva, di spaesamento di fronte a una disfatta che, seppur ascrivibile a una personalità controversa come Giuliano, colpiva il mondo romano. Ancora una volta vittima dei duces bellorum sassanidi, qui raffigurati nelle parvenze di una figura duplicemente condannabile, sia in base ai canoni pagano-ellenistici che a quelli cristiani, in un crescendo di epiteti (persiano, mago, istrione) che ricordavano altre espressioni dello sciovinismo latino - consueti nella tradizione paremiografica, tipo punica fides o graeca fides - verso soggetti etnici ritenuti crudeli, infidi e fraudolenti, come ci ha vividamente descritto Ammiano - contemporaneo degli Acta Archelai - in molti passaggi della sua opera, parlando di usi, costumi e indole dei sassanidi. ${ }^{43}$

\section{BiBLIOGRAFIA}

Adam, A. (1969), Texte zum Manichäismus, Berlin.

Barb A.A. (1975), La sopravvivenza delle arti magiche, in: A. Momigliano (a cura di), Il conflitto tra paganesimo e cristianesimo nel secolo IV, Torino: 113-137.

BeDuhn J., Mirecki P. (eds.) (2007), Frontiers of Faith: The Christian Encounter with Manichaeism in the Acts of Archelaus, Leiden-Boston.

Beeson, C.H. (1906), Hegemonius Acta Archelai, Leipzig.

Brown, P. (1999), Il filosofo e il monaco: due scelte tardoantiche, in: A. Giardina, A. Schiavone (a cura di), Storia di Roma, Torino: 661-678.

Bucci, O. (1978), Il matrimonio fra consanguinei (khvêtûkdâs) nella tradizione giuridica delle genti iraniche, Apollinaris 51: 291-319.

Cameron, A. (2013), The Cost of Orthodoxy, Leiden.

Coloru, O. (2017), L'imperatore prigioniero. Valeriano, la Persia e la disfatta di Edessa, Bari-Roma.

Coyle, J.K. (2004), Foreign and Insane: Labelling Manichaeism in the Roman Empire, Studies in Religion/Sciences Religieuses 33/2: 217-234.

Dignas, B., Winter, E. (2007), Rome and Persia in Late Antiquity: Neighbours and Rival, Cambridge.

Drijvers, J.W. (2006), Ammianus Marcellinus' Image of Sasanian Society, in: J. Wiesehöfer, Ph. Huyse (hrsg.), Ërān und Anērān. Studien zu den Beziehungen zwischen dem Sasanidenreich und der Mittelmeerwelt, München: 45-69.

Fox, G., Sheldon, J., Lieu, S.N.C. (2010), Greek and Latin Sources ou Manichean Cosmogony and Ethics, Turnhout-Sydney.

Gardner, I. (1995), The Kephalaia of the Teacher, Leiden-Boston.

Gardner, I., Lieu, S.N.C. (2004), Manichaean Texts from the Roman Empire, Cambridge-New York.

Gignoux, Ph. (1993), Contacts culturels entre manichéisme et mazdéisme: quelques exemples significatifs, Studia Orientalia 70: 65-73.

Ginzburg, C. (1995), Storia notturna. Una decifrazione del sabba, 2nda ed., Torino.

Gnoli, G. (1996), Il pericolo persiano: Ardashir e Shapur I, in: Storia della società italiana, vol. 3: La crisi del principato e la società imperiale, Milano: 399-433.

\footnotetext{
${ }^{42}$ Cf. Mazza 2005, 132-145, su questi avvenimenti bellici e diplomatici.

${ }^{43}$ Si veda Drijvers 2006 per una dettagliata investigazione dei passi di Ammiano relativi ai Sassanidi.
} 
Gnoli, G. (a cura di) con l'assistenza di A. Piras (2003), Il Manicheismo, vol. 1: Mani e il Manicheismo, Milano.

Gnoli, G. (a cura di) con l'assistenza di A. Piras (2006), Il Manicheismo, vol. 2: Il mito e la dottrina. I testi manichei copti e la polemica antimanichea, Milano.

Gnoli, G. (a cura di) con l'assistenza di A. Piras (2008), Il Manicheismo, vol. 3: Il mito e la dottrina. Testi manichei dell'Asia Centrale e della Cina, Milano.

Graf, D.F. (1984), Medism: The Origin and Significance of the Term, JHS 104: 15-30.

Hall, E. (1989), Inventing the Barbarian: Greek Self-Definition through Tragedy, Oxford-New York.

Hornblower (ed.), Greek Historiography, Oxford: 233-247.

Klíma, O. (1957), War Mānī wirklich lahm? (Ein Beitrag zur Geschichte des Odium theologicum), Archiv Orientální 25: 384-387.

Klíma, O. (1968), Wie sah die persische Geschichtsschreibung in der vorislamischen Periode aus?, Archiv Orientální 36: 213-230.

Lieu, S.N.C. (1999), Manichaeism in Mesopotamia \& the Roman East, $2^{\text {nd }}$ ed., Leiden-Boston.

Lieu, S.N.C. (2000), A New Figurative Representation of Mani?, in: R.E. Emmerick et al. (hrsg.), Studia Manichaica IV, Berlin: 380-386.

Magris, A. (2016), Plotino alla guerra, in: V. Formentin et al. (a cura di), Lingua, letteratura e umanità. Studi offerti dagli amici ad Antonio Daniele, Padova: 29-41.

Marasco, G. (1993), Costantino e le uccisioni di Crispo e Fausta (326 d.C.), RFIC 121: 297-317.

Mazza, M. (2005), Bisanzio e Persia nella tarda antichità. Note su guerra e diplomazia nella seconda metà del IV secolo D.C., in: M. Mazza, Cultura, guerra e diplomazia nella tarda antichità. Tre studi, Catania: 121-167.

Minale, V.M. (2013), Strategius Musonianus, un funzionario poliglotta?, in: C. Cascione et al. (a cura di), Modelli di un multiculturalismo giuridico: il bilinguismo nel mondo antico. Diritto, prassi, insegnamento, Napoli: 649-673.

Minale, V.M. (2014), Costantino, Strategio Musoniano e i Manichei. Ancora su Amm. Marc. Res Gestae 15.13.2, in: S. Randazzo (a cura di), Religione e Diritto Romano. La cogenza del rito, Le Tricase: $333-356$.

Muccioli, F. (2012), La testa mozza di Crasso (Plut., Crass. 32-33). A proposito di un libro recente sulla battaglia di Carre, Electrum 9: 167-178.

Neri, V. (2004), Vestito e corpo nel pensiero dei Padri tardoantichi, Antiquité Tardive 12: 223-230.

Panaino, A. (2004), La Chiesa di Persia e l'impero sasanide. Conflitto e integrazione, in: Cristianità d'Occidente e cristianità d'Oriente (secoli VI-XI), Spoleto: 765-863.

Panaino, A. (2010), I Magi in Occidente, in: G. M. Cazzaniga (a cura di), Storia d'Italia, Annali 25, Torino: 49-76.

Panaino, A. (2011), Erodoto, i Magi e la storia religiosa iranica, in: R. Rollinger et al. (hrsg.), Herodot und das Persische Weltreich / Herodotus and the Persian Empire, Wiesbaden: 43-70.

Paratore, E. (1966), La Persia nella letteratura latina, in: La Persia e il mondo greco-romano, Roma: 505-558.

Piras, A. (2005), Manichaeus claudicans. La zoppaggine dell'eretico e del taumaturgo, in: M. Bernardini, N.L. Tornesello (a cura di), Scritti in onore di Giovanni M. D'Erme, Napoli: 869-887.

Piras, A. (2013), La politica con i sasanidi. Conflitti, diplomazia e nuove problematiche religiose, in: Costantino I. Enciclopedia costantiniana sulla figura e l'immagine dell'imperatore del cosiddetto Editto di Milano 313-2013, vol. I, Roma: 415-430.

Questa, C. (1989), Il morto e la madre. Romei e Persiani nelle 'Storie' di Agatia, Lares 3: 375-405.

Ries, J. (1979), Théologie solaire manichéenne et culte de Mithra, in: U. Bianchi (éd.), Mysteria Mithrae, Leiden-Roma: 761-775.

Riggi, C. (1967), Epifanio contro Mani, Roma.

Roberto, U. (2014a), Diocleziano, Roma.

Roberto, U. (2014b), Beatissimum saeculum: Diocleziano, la Persia e la persecuzione dei manichei, in: A. Marcone et al. (a cura di), Tolleranza religiosa in età tardoantica, $I V-V$ secolo, Cassino: 179-214. 
Schneider, R.M. (2006), Orientalism in Late Antiquity: The Oriental in Imperial and Christian Imagery, in: J. Wiesehöfer, Ph. Huyse (hrsg.), Ērān ud Anērān. Studien zu den Beziehungen zwischen dem Sasanidenreich und der Mittelmeerwelt, München: 241-278.

Scopello, M. (2008), Un témoin de la controverse religieuse entre chrétiens et manichéens aux frontières de l'Iran: les Acta Archelai, in: Ch. Jullien (éd.), Controverses des chrétiens dans l'Iran sassanide, Paris: $147-168$.

Spawforth, A. (1994), Symbol of Unity? The Persian-Wars Tradition in the Roman Empire, in: S. Hornblower (ed.), Greek Historiography, Oxford: 233-247.

Tantillo, I. (1997), La Prima Orazione di Giuliano a Constanzo: Introduzione, Traduzione e Commento, Roma.

Tardieu, M. (1981), Le manichéisme, Paris.

Traina, G. (2010), La resa di Roma: 9 giugno 53 a.C., battaglia a Carre, Roma-Bari.

Tuplin, C.J. (1997), Medism and its Causes, Transeuphratène 13: 155-185.

Vannicelli, P. (2007), L'epoca delle guerre persiane, in: M. Giangiulio (a cura di), Storia d'Europa e del Mediterraneo. Il mondo antico 1. Sezione II: La Grecia, vol. III, Roma: 561-598.

Vermes, M., Lieu S.N.C., Katz, K. (2001), Hegemonius Acta Archelai (The Acts of Archelaus), Lovanii. Villey, A. (1985), Alexandre de Lycopolis. Contre la doctrine de Mani, Paris. 